\author{
Karla Diamantina de Araújo Soares
}

\title{
Sistemática do gênero Scyliorhinus Blainville, 1816 (Chondrichthyes: Carcharhiniformes: Scyliorhinidae)
}

\section{Systematics of the genus Scyliorhinus Blainville, 1816 (Chondrichthyes: Carcharhiniformes: Scyliorhinidae)}

\begin{abstract}
Resumo
O gênero Scyliorhinus pertence a família Scyliorhinidae, a mais diversa dentre os tubarões, e a subfamília Scyliorhininae, juntamente com Cephaloscyllium e Poroderma. Há atualmente 16 espécies válidas para Scyliorhinus, embora muitas questões sejam levantadas sobre a definição e composição do gênero, principalmente a respeito da identidade de $S$. canicula. Além disso, o longo histórico taxonômico de rearranjos e descrições inacuradas de muitas espécies tem contribuído para identificações errôneas dos espécimes e falta de informação sobre a distribuição geográfica e os caracteres diagnósticos de muitas espécies. Este estudo teve como objetivo revisar a taxonomia do gênero e estimar a distribuição geográfica de todas as suas espécies. Adicionalmente, as relações de parentesco das espécies de Scyliorhinus dentro da subfamília Scyliorhininae foram investigadas. Espécimes de todas as espécies de Scyliorhinus foram examinados bem como espécimes de quatro espécies de Cephaloscyllium spp., duas espécies de Poroderma spp. e representantes de quase todos os demais gêneros de cações-gato. Um estudo morfológico detalhado, incluindo morfologia externa, anatomia interna, morfometria e dados merísticos, foi realizado. A partir deste estudo, 77 caracteres morfológicos e três merísticos foram compilados em uma matriz. Análise de parcimônia foi empregada para
\end{abstract}


gerar hipóteses de relações de parentesco usando o programa TNT 1.1. Asymbolus analis foi usado para enraizar o cladograma. As análises de relações de parentesco baseadas em buscas com pesagem implícita ( $k=3 ; 500$ réplicas e 100 árvores salvas por repl.) resultaram em sete cladogramas igualmente mais parcimoniosos com 231 passos, com um CI de 0,42 e um RI de 0,69. A monofilia da subfamília Scyliorhininae é suportada com base na ausência do músculo depressor palpebrae nictitans assim como de uma bifurcação na borda anterior da cartilagem basihial e de uma cartilagem marginal dorsal acessória nos clásperes, um número inferior de cartilagens extrabranquiais ventrais, uma cobertura dérmica terminal mais longa no clásper e sifões do clásper curtos. A monofilia de Scyliorhinus é suportada pela presença de uma aba projetada sobre o lábio superior assim como por um apron pélvico e uma maior extensão deste. Scyliorhinus é proposto como grupo-irmão de Cephaloscyllium, hipótese sustentada pela presença de processos rastriformis iguais em tamanho e uma projeção medial da barra coracoide bem desenvolvida. As relações de parentesco entre todas as espécies de Scyliorhinus são apresentadas pela primeira vez. Neste estudo, 16 espécies são reconhecidas: Scyliorhinus boa, S. cabofriensis, S. canicula, S. capensis, S. cervigoni, S. comoroensis, S. duhamelli, S. garmani, S. haeckelii, S. hesperius, S. meadi, S. retifer, S. stellaris, S. torazame, S. torrei, e $S$. ugoi. As principais mudanças taxonômicas incluem a revalidação de $S$. duhamelii e a sinonimização de $S$. tokubee com $S$. torazame. A informação sobre a distribuição geográfica foi atualizada para a maioria das espécies, especialmente para aquelas com distribuições amplas (S. canicula, S. haeckelii, S. retifer e S. stellaris). Coloração, contagens de vértebras, morfologia do clásper, tamanho de maturidade e configuração das abas nasais consistem nos caracteres mais úteis para a distinção entre essas espécies.

\begin{abstract}
The genus Scyliorhinus belongs to the family Scyliorhinidae, the most diverse family of sharks, and to the subfamily Scyliorhininae, with Cephaloscyllium and Poroderma. There are currently 16 valid species for Scyliorhinus, however many questions are raised concerning the definition and composition of the genus, mainly regarding the identity of $S$. canicula. Furthermore, the long history of taxonomic rearrangements and inaccurate descriptions of many species have contributed to misidentification of specimens and lack of information on the distribution range and diagnostic characters of many species. This study aimed to review the taxonomy of the genus and to estimate the geographic distribution of all its species. In addition, the phylogenetic
\end{abstract}


relationships of the Scyliorhinus species within the subfamily Scyliorhininae was investigated. Specimens of all Scyliorhinus species were examined as well as specimens of four species of Cephaloscyllium spp., two species of Poroderma spp. and representatives of almost all other catshark genera. A detailed morphological study, including external morphology, internal anatomy, morphometry and meristic data, were performed. From this study, a total of 77 mophological and three meristic characters were compiled into a matrix. Parsimony analysis was employed to generate hypotheses of phylogenetic relationships using the TNT 1.1 computer program. Asymbolus analis was used to root the cladogram. The analysis of the phylogenetic relationships based on implied weighting $(k=3 ; 500$ replications and 100 trees saved per repl.) resulted in seven equally most parsimonious cladograms with 231 steps, with a CI of 0.42 and an RI of 0.69. The monophyly of the subfamily Scyliorhininae is supported on the basis of the absence of the muscle depressor palpebrae nictitans as well as of the bifurcation on the leading edge of basihial cartilage and accessory marginal cartilage on the claspers, a lower number of ventral extra branchial cartilages, a greater extension of the dermal terminal cover on the clasper and short clasper siphons. The monophyly of Scyliorhinus is supported on the basis of the presence of a projecting tab at upper lip margin as well as of a pelvic apron and a greater extension of it. Scyliorhinus is proposed as the sister group of Cephaloscyllium, based on the presence of processus rastriformis similar in length and a welldeveloped medial projection of the coracoid bar. The phylogenetic relationships among Scyliorhinus species are presented for the first time. In this study, 16 species are recognized: Scyliorhinus boa, S. cabofriensis, S. canicula, S. capensis, S. cervigoni, S. comoroensis, S. duhamelli, S. garmani, S. haeckelii, S. hesperius, S. meadi, S. retifer, S. stellaris, S. torazame, S. torrei, and S. ugoi. The main taxonomic decisions include the resurrection of S. duhamelii and the synonimization of $S$. tokubee with S. torazame. The information of the geographic distribution was updated for most species, especially for those with wide ranges ( $S$. canicula, S. haeckelii, S. retifer and S. stellaris). Coloration, vertebral counts, clasper morphology, maturity size and configuration of nasal flaps are the most useful characters to distinguish among species. 


\section{Introdução}

Os tubarões popularmente conhecidos como cações-gato pertencem à família Scyliorhinidae, a mais diversa da ordem Carcharhiniformes, compreendendo 17 gêneros e aproximadamente, 160 espécies válidas (Compagno et al., 2005; Ebert et al., 2013). Estes tubarões são caracterizados por apresentar corpo delgado, olhos caracteristicamente alongados horizontalmente, nadadeiras dorsais mais posteriores em relação às pélvicas, nadadeiras peitorais triangulares e paralelas ao eixo do corpo, sulcos pré-caudais ausentes e lobo inferior da nadadeira caudal pouco desenvolvido (Springer, 1979; Compagno, 1984, 1988a).

Os cações-gato vivem associados ao substrato marinho, desde regiões costeiras até a borda da plataforma continental, chegando até profundidades de $2.000 \mathrm{~m}$. O tamanho máximo, quando adulto, varia entre $30 \mathrm{~cm}$ e $1,6 \mathrm{~m}$, com comprimento total médio de 70-80 cm. A estratégia de desenvolvimento do embrião dá-se por oviparidade, na qual a fêmea retém o ovo fertilizado no útero, depositando-o no substrato marinho depois de certo período. Alimentam-se primariamente de invertebrados e pequenos peixes (Bigelow \& Schroeder, 1948; Springer, 1966, 1979; Compagno et al., 2005; Ebert et al., 2013).

O gênero Scyliorhinus Blainville, 1816 é composto por 16 espécies viventes atualmente reconhecidas, cuja distribuição geográfica abrange todos os mares, de águas frias até tropicais, excetuando-se a Antártica, leste e sudoeste do Oceano Pacífico, e sudeste do Oceano Índico (Compagno, 1988a; Compagno et al., 2005; Ebert et al., 2013). Contribuições ao conhecimento sobre biologia, evolução e anatomia dos tubarões foram e continuam sendo realizadas, graças à diversos estudos com a espécie $S$. canicula, considerada por muitos um organismo modelo, bem como Squalus acanthias, para estudos em elasmobrânquios. Manuais de anatomia comparada contendo descrições de S. canicula são maciçamente encontrados na literatura (Parker \& Haswell, 1897; Bourne, 1902; Parker, 1914; Kerr, 1921; O’Donoghue, 1921; de Beer, 1929; Yapp, 1958), assim como estudos sobre experimentos em cativeiro, reprodução, genética, fisiologia, comportamento, entre outros (Day, 1880; Ford, 1921; Capapé et al., 2000). Entretanto, questões acerca da taxonomia e das relações de parentesco desta espécie e suas congêneres com os demais táxons da família permeiam a literatura, as quais serão detalhadas ao longo deste estudo. 


\section{Taxonomia do gênero Scyliorhinus Blainville, 1816}

O gênero Scyliorhinus, englobando os gêneros nominais sinônimos Scyllium Cuvier, 1816, Scylliorhinus Blainville, 1825, Catulus Smith, 1838 e Catulus Valmont de Bomare, 1769, possui uma longa história taxonômica, intimamente relacionada com a história da família Scyliorhinidae. Referências aos cações-gato deste gênero são encontradas na literatura desde os trabalhos de Aristóteles (a.c. século IV; Rondelet, 1554; Hoffman \& Jordan, 1892) e em trabalhos pré-lineanos (Rondelet, 1554; Salviani, 1554; Gesner, 1586; Artedi, 1738; Gronovius, 1756). Ilustrações e breves descrições sobre Scyliorhinus canicula (Linnaeus, 1758) e S. stellaris (Linnaeus, 1758), as primeiras espécies reconhecidas para o gênero, são encontradas em trabalhos clássicos para o estudo de peixes, principalmente em catálogos das espécies conhecidas para o continente europeu (Artedi, 1738; Klein, 1742; Linnaeus, 1758; Salviani, 1766; Bruchinii, 1768; Berkenhout, 1769; Bloch, 1782-1795; Bloch, 1796; Pennant, 1812; Lacépède, 1830). Desde então, diversas alterações ocorreram com relação às espécies integrantes do gênero e à definição de seus caracteres diagnósticos.

O gênero Scyliorhinus foi proposto por Blainville (1816), baseando-se nos caracteres dentes tricuspidados, presença de espiráculos, região caudal longa e padrão de coloração variado, e posicionando-o dentro do táxon Squalus. Posteriormente, Müller \& Henle (183841) incluíram o gênero Scyllium (= Scyliorhinus) dentro da família Scyllia (= Scyliorhinidae), a qual seria caracterizada pelos mesmos caracteres mencionados por Blainville (1816) para Scyliorhinus. Além destes caracteres, Müller \& Henle (1838-41) listaram a posição mais posterior da $1^{\text {a }}$ nadadeira dorsal em relação às pélvicas, característica essa que estaria relacionada a uma região caudal longa. Autores subsequentes seguiram tais propostas de classificação, embora diferentes nomes tenham sido utilizados para se referir ao gênero (Cuvier, 1817, 1829, 1834; Duperrey, 1830; Jenyns, 1835; Bostock, 1837; Parnell, 1838; Swainson, 1839; Agassiz 1833-1845; Hamilton, 1854; Duméril,1865; Bocage \& Capell, 1866; Günther, 1870; Day, 1878; Donderlein, 1879-1880; Day, 1880-84; Moreau, 1886).

Os diferentes nomes atribuídos à Scyliorhinus estão intimamente relacionados ao histórico de realocações de espécies em outros gêneros e às diferenças encontradas entre a espécie-tipo, S. canicula, e suas congêneres (Gill, 1862; Jordan \& Evermann, 1896; Danois, 1913; Garman, 1913). Com exceção dos gêneros Figaro e Galeus (também citado como Pristiurus), cuja as espécies foram, desde o início, distinguidas pela presença de uma crista caudal de dentículos dérmicos especializadas, espécies classificadas atualmente nos demais 
gêneros da família Scyliorhinidae já foram agrupadas em Scyliorhinus (Blainville, 1816; Müller \& Henle, 1838-41; Bennett, 1830; Cuvier, 1834; Duméril, 1853; Günther, 1870; Hilgendorf, 1904; Regan, 1908; Tanaka, 1909; Barnard, 1925; Fowler, 1934; Whitley, 1939; Springer, 1966). Além disso, espécies atualmente pertencentes à família Orectolobidae Brachaelurus, Hemiscyllium, Orectolobus, Stegostoma - também já foram alocadas em Scyliorhinus, devido a semelhanças na região nasoral entre $S$. canicula e Orectolobiformes (Blainville, 1816; Cuvier, 1824; Günther, 1870).

Em diversos trabalhos, o nome Scyliorhinus (ou Scylliorhinus) foi utilizado para se referir às espécies com abas nasais anteriores próximas entre si, enquanto que Catulus ou Scyllium referiam-se àquelas com abas nasais separadas (Gill, 1862; Jordan \& Evermann, 1896; Danois, 1913). Até que em seu trabalho clássico sobre tubarões e raias, Garman (1913) propôs a adoção de Catulus para todas as espécies de Scyliorhinus até então conhecidas, argumentando que as inúmeras e sucessivas realocações e propostas de outros gêneros a partir de espécies anteriormente atribuídas à Scyliorhinus impossibilitariam a manutenção do nome do mesmo. No entanto, o nome Catulus Valmont, 1769 foi rejeitado pela Comissão Internacional em Nomenclatura Zoológica, Opinião 89 (1925), por não seguir as regras da nomenclatura binomial.

Bigelow \& Schroeder (1948) propuseram uma classificação com apenas sete espécies no gênero Scyliorhinus (S. boa, S. canicula, S. capensis, S. retifer, S. stellaris, S. torazame e, S. torrei), excluindo espécies pertencentes aos demais gêneros. Autores subsequentes seguiram tal proposta, por vezes acrescentando novas spécies ou sinonimizando as existentes (Springer, 1966, 1979; Compagno, 1984; Compagno, 1988b; Shirai et al., 1992; Compagno et al., 2005; Ebert et al., 2013; Soares et al., 2015, 2016).

Atualmente, 16 espécies válidas são reconhecidas em Scyliorhinus, sendo elas: S. boa Goode \& Bean, 1896, S. cabofriensis Soares, Gomes \& de Carvalho, 2016, S. canicula, S. capensis (Muller \& Henle, 1838), S. cervigoni Maurin \& Bonnet, 1970, S. comoroensis Compagno, 1988, S. garmani (Fowler, 1934), S. haeckelii (Miranda Ribeiro, 1907), S. hesperius Springer, 1966, S. meadi Springer, 1966, S. retifer (Garman, 1881), S. stellaris, S. tokubee Shirai, Hagiwara \& Nakaya, 1992, S. torazame (Tanaka, 1908), S. torrei Howell Rivero, 1936 e, S. ugoi Soares, Gadig \& Gomes, 2015. Vinte e uma espécies nominais foram propostas na literatura, com quatro nomes adisponíveis atualmente para espécies de Scyliorhinus (Springer, 1979; Compagno, 1988a; Soares et al., 2016). 
O caráter mais utilizado para a identificação e separação das espécies de Scyliorhinus é o padrão de coloração apresentado pelos indivíduos. Tal fato pode ser confirmado pelos trabalhos de Springer (1966, 1979), que apesar de citar outros caracteres, tais como, morfométricos, de dentição, dados merísticos e de clásperes, considerou majoritariamente características de coloração em suas chaves taxonômicas. Outro exemplo está presente no trabalho de Springer \& Sadowsky (1970), no qual os autores dividiram S. retifer em quatro subespécies e descreveram uma nova subespécie, S. retifer besnardi, considerando apenas a coloração dos exemplares examinados. Entretanto, variações individuais, dimorfismo sexual e mudanças ontogenéticas influenciam no padrão de coloração apresentado pelos indivíduos, podendo dificultar a identificação das espécies (Soares et al., 2015, 2016). Além disso, as chaves de identificação presentes na literatura são bastante ambíguas e não apresentam dados detalhados sobre o padrão de coloração, dificultando a identificação inequívoca dos espécimes. Por exemplo, $S$. torrei e $S$. hesperius não seriam diferenciados pela chave de identificação apresentada por Springer $(1966,1979)$.

As únicas espécies do gênero facilmente identificadas são $S$. canicula, por seus sulcos nasorais característicos, e S. retifer, por seu padrão de coloração reticulado (Compagno et al., 2005; Ebert et al., 2013). Trabalhos taxonômicos contendo dados detalhados de medidas, morfologia externa, clásperes e comparações com outras espécies do gênero só existem para cinco espécies (S. cabofriensis, S. comoroensis, S. haeckelii, S. tokubee e, S. ugoi) (Compagno, 1988b; Shirai et al., 1992; Soares et al., 2015, 2016). Outras espécies foram descritas com base em exemplares imaturos, como por exemplo, S. hesperius e $S$. meadi; somente os holótipos são conhecidos de $S$. garmani e $S$. comoroensis, e os tipos de quatro espécies ( $S$. canicula, $S$. cervigoni, S. stellaris e S. torazame) são desconhecidos ou foram perdidos (Springer, 1966, 1979; Compagno, 1988b; Van der Laan et al., 2014).

Apesar do hábito de se associar ao substrato e de não realizar migrações a grandes distâncias, algumas espécies, tais como S. canicula, que ocorre nas águas dos mares do Norte, Mar Mediterrâneo, Atlântico Norte Ocidental e Noroeste do continente africano (Compagno, 1984; Compagno et al., 2005), S. haeckelii, do norte da Argentina até a Colômbia (Springer \& Sadowsky, 1970; Compagno, 1984; Compagno et al., 2005) e, S. retifer, do Golfo do México até o norte dos Estados Unidos (Compagno, 1984; Compagno et al., 2005), apresentam áreas de distribuição consideravelmente grandes. Tais distribuições levantam questões a respeito da identidade dos indivíduos e dos limites de variação das espécies (Litvinov, 2003; RodríguezCabello et al., 2004; Bacilieri, 2005; Soares et al., 2016). 
Descrições mais detalhadas sobre todas as espécies de Scyliorhinus, principalmente sobre a morfologia externa, neurocrânios e clásperes, são necessárias para que uma comparação mais efetiva seja realizada e mais caracteres morfológicos possam ser considerados em análises filogenéticas, contribuindo para diminuir os erros e dúvidas na identificação dos indivíduos e propiciando um conhecimento maior a respeito destes tubarões que possuem padrões de coloração tão variados.

\section{Filogenia do gênero Scyliorhinus e demais gêneros da subfamília Scyliorhininae}

Hip2óteses divergentes sobre a classificação dos cações-gato estão presentes na literatura e dividem as opiniões de diversos autores (Compagno, 1988a; Iglésias et al., 2005; Human et al., 2006; Naylor et al., 2012; Van der Laan et al., 2014; Nelson et al., 2016). Analisando dados morfológicos, Compagno (1998a) propôs o agrupamento de 17 gêneros na família Scyliorhinidae, seguindo o arranjo tradicional para o grupo. Posteriormente, Iglésias et al. (2005), analisando dados moleculares, hipotetizaram a parafilia da família Scyliorhinidae e propuseram a redefinição de Scyliorhinidae e a alocação de 10 gêneros em uma nova família, Pentanchidae. Segundo os autores, Scyliorhinidae e Pentanchidae poderiam ser diferenciados morfologicamente pela presença/ausência da crista supraorbital no neurocrânio.

Naylor et al. (2012), em uma análise incluindo um número maior de táxons composta por 55 espécies de 15 gêneros da família Scyliorhinidae e analisando dados moleculares, propuseram três linhagens distintas dentro desta. Os autores ressaltaram ainda o posicionamento de espécies pertencentes a outras famílias entre os táxons de Scyliorhinidae, refutando o monofiletismo da família. Desde então, nenhuma análise cladística estrita para a família Scyliorhinidae utilizando dados morfológicos foi realizada. No entanto, independentemente da classificação e arranjo considerados, o gênero Scyliorhinus continua pertencendo à família Scyliorhinidae.

Compagno (1988a) reuniu o gênero Scyliorhinus, juntamente com Poroderma e Cephaloscyllium, dentro da subfamília Scyliorhininae, seguindo a proposta de Gill (1862) e baseando-se em caracteres provenientes de músculos e neurocrânio. Herman et al. (1990) propuseram o mesmo agrupamento, baseando-se em caracteres dentários. Posteriormente, análises moleculares (genes nucleares e mitocondriais) recuperaram o monofiletismo desta subfamília (Iglésias et al., 2005; Human et al., 2006; Naylor et al., 2012), embora as relações 
entre os gêneros constituintes tenham apresentado divergências entre as propostas com dados morfológicos e moleculares (Compagno, 1988a; Naylor et al., 2012).

Questionamentos com relação ao monofiletismo de Scyliorhinus são encontrados em diversos trabalhos, principalmente no que se refere às relações de parentesco entre $S$. canicula e as demais espécies do gênero (Springer, 1966, 1979; Compagno, 1988a). Tal espécie apresenta algumas características estruturais na região nasoral que a distingue de seus congêneres, tais como a presença de sulcos nasorais e abas nasais anteriores encobrindo o lábio superior (Springer, 1966, 1979). Estas características também seriam encontradas em Atelomycterus e Haploblepharus, sendo resultantes de uma provável convergência evolutiva (Compagno, 1988a). Tais diferenças seriam, segundo Springer (1979), suficientemente grandes e únicas na família para garantir a alocação das demais espécies de Scyliorhinus em um gênero distinto, assim como proposto por Jordan \& Evermann (1896) e Danois (1913). Compagno (1988a) sugeriu a adoção do nome Betascyllium Leigh-Sharpe, 1926b, caso tal rearranjo fosse necessário. Bell (1993), por sua vez, ressaltou a importância de analisar cautelosamente os caracteres da região nasoral, examinando-se uma quantidade representativa de táxons para melhor compreensão da evolução destes caracteres dentro da família Scyliorhinidae.

O único caráter exclusivo de Scyliorhinus seria a ausência de um sulco labial superior concomitante com a presença de um estreito sulco inferior (Compagno, 1988a). A presença de uma aba projetada sobre o sulco labial inferior, citada por alguns autores como um caráter relevante para a identificação das espécies de Scyliorhinus (Bigelow \& Schroeder, 1948; Springer, 1966, 1979), não foi considerada como uma sinapomorfia para o gênero por Compagno (1988a). Além disso, de acordo com alguns autores (Springer, 1979; Compagno, 1988a), a configuração dos sulcos labiais em Poroderma e em algumas espécies de Cephaloscyllium poderia ser facilmente confundida com a configuração apresentada por Scyliorhinus, visto que nestes dois gêneros tais estruturas são pouco desenvolvidas ou ausentes e os limites seriam mal definidos (Compagno, 1988a).

Portanto, faz-se necessário um maior detalhamento e a reinterpretação de caracteres morfológicos, tais como sulcos labiais, abas nasais, posição relativa das nadadeiras e padrões de coloração, bem como o estudo comparativo de estruturas anatômicas (e.g. neurocrânio, arcos viscerais, musculatura e clásperes) para a compreensão da variação interespecífica existente em Scyliorhinus assim como das relações de parentesco entre suas espécies e os demais táxons de Scyliorhininae. 


\section{Conclusões}

1. Dezesseis espécies de Scyliorhinus são consideradas válidas no presente trabalho, todas já descritas previamente. As principais mudanças taxonômicas incluem a revalidação de S. duhamelii (Garman, 1913) e a sinonimização de S. tokubee Shirai et al., 1992 com S. torazame (Tanaka, 1908);

2. Dados de distribuição geográfica de todas as espécies são atualizados, com base na reidentificação dos espécimes, reinterpretação dos dados de localidade e o aumento do número de espécimes examinados;

3. A subfamília Scyliorhininae é considerada monofilética, sendo esta hipótese sustentada com base na ausência do músculo depressor palpebrae nictitans, ausência de bifurcação na borda anterior da cartilagem basihial, presença de três cartilagens extrabranquiais ventrais, ausência da cartilagem marginal dorsal acessória no clásper, cobertura dérmica terminal estendendo-se por pelo menos $1 / 3$ da glande do clásper e, sifão do clásper curto;

4. Scyliorhinus é proposto como grupo-irmão de Cephaloscyllium, hipótese sustentada com base na presença de processos rastriformis laterais do mesmo tamanho que os demais e uma projeção medial da barra coracoide bem desenvolvida;

5. O gênero Scyliorhinus é proposto como monofilético, sendo esta hipótese sustentada com base na presença de uma aba projetada sobre o lábio superior, presença de um apron pélvico e extensão deste correspondendo à mais da metade do comprimento das margens internas das pélvicas em machos;

6. As relações de parentesco de todas as dezesseis espécies de Scyliorhinus e demais Scyliorhininae são apresentadas pela primeira vez na literatura. Com relação à resolução obtida, $S$. boa e $S$. retifer compõem um clado proximamente relacionado à todas as demais espécies do gênero; S. cabofriensis, S. cervigoni, S. haeckelii e S. ugoi formam um clado sustentado pela presença de uma cartilagem terminal dorsal 2 reduzida no clásper e, S. canicula, S. capensis, S. duhamelii, S. torazame e, S. torrei 
hipotetizados como proximamente relacionados pela presença de um apron pélvico bem desenvolvido em machos.

\section{Referências Bibliográficas}

Agassiz, L. (1833-1845) Recherches sur les poissons fossiles. Tome III. Neuchatel, Petitpierre, 383pp.

Akhilesh, K.V., Bineesh, K.K., Gopalakrishnan, A., Jena, J.K., Basheer, V.S. \& Pillai N.G.K. (2014) Checklist of Chondrichthyans in Indian waters. Journal of the Marine Biological Association of India, 56 (1), 109-120.

Azouz, A. \& Capapé, C. (1971) Les relations alimentaires entre les selaciens et le zoobenthos des cotes nord de la Tunisie. Bull. Inst. Océanogr. Pêche, Salammbô, 2 (2), 121-130.

Artedi, P. (1738) Ichthyologia sive opera omnia de piscibus, scilicet: Bibliotheca ichthyologica. Philosophia ichthyologica. Genera piscium. Synonymia specierum. Descriptiones specierum. Omnia in hoc genere perfectiora, quam antea ulla. Lugduni Batavorum, apud Conradum Wishof, 118 pp.

Bacilieri, S. (2005) Revisão taxonômica do gênero Scyliorhinus Blainville, 1816 (Carcharhiniformes, Scyliorhinidae), nas regiões sudeste e sul do Brasil. Unpubl. PhD thesis, Universidade Estadual Paulista 'Júlio Mesquita Filho', Rio Claro, 82pp.

Balfour, F.M. (1881) On the development of the skeleton of the paired fins of Elasmobranchii, considered in relation to its bearings on the nature of the limbs ot the Vertebrata. Proceedings of the Zoological Society of London, 1881, 656-671.

Bänarescu, P. (1969) Cyclostomata si Chondrichthyes. Fauna Repub. Soc. Romênia 12, 106pp.

Barnard, K.H. (1925) A monograph of the marine fishes of South Africa. Part I (Amphioxus, Cyclostomata, Elasmobranchii, and Teleostei - Isospondyli to Heterosomata). Annals of the South African Museum, 21 (1), 1-418. 
Barnard, K.H. (1947) A Pictorial Guide to South African Fishes. Marine and Freshwater. Maskew Miller, Cape Town, 226pp.

Bass, A.J. (1973) Analysis and description of variation in the proportional dimensions of scyliorhinid, carcharhinid and sphyrnid sharks. South African Association for Marine Biological Research, Oceanographic Research Institute, Investigational Report, 32, 28 pp.

Bass, A.J. (1986) Families Chlamydoselaehidae. Scyliorhinidae. Lamnidae, Alopiidae. In: Smith, M. M. \& Heemstra, P.C. (Eds.), Smiths' Sea Fishes.; Macmillan: Johannesburg, pp. 88-95, 98-102.

Bass, A.J, D'Aubrey, J.D. \& Kistnasamy, N. (1975) Sharks of the east coast of Southern Africa. II. The families Scyliorhinidae and Pseudotriakidae. Investigational Report Oceanographic Research Institute, 37, 1-64.

de Beer, G.R. (1928) Vertebrate Zoology: an introduction to the comparative anatomy, embryology, and evolution of chordate animals. The Macmillan Company, New York, 505pp.

Bell, M.A. (1993) Convergent Evolution of Nasal Structure in Sedentary Elasmobranchs. Copeia, 1993 (1), 144-158.

Bennet, E.T. (1830) Class Pisces. In: Memoir of the Life and Public Services of Sir Thomas Stamford Raffles. Lady Stamford Raffles, pp. 686-694.

Berkenhout, J. (1769) Outlines of the natural history of Great Britain and Ireland, containing a systematic arrangement and concise description of all the animals, vegetables and fossils which have hitherto been discovered in these kingdoms. vol. 1. London, 233 pp.

Bianchi, G., Carpenter, K.E., Roux, J.P., Molloy, F.J., Boyer, D. \& Boyer, H.J. (1999) FAO species identification field guide for fishery purposes. The living marine resources of Namibia. FAO, Rome, 250pp. 
Bigelow, H.B. \& Schroeder W.C. (1948) Fishes of the Western North Atlantic. Part I. Lancelets, Cyclostomes and Sharks. Yale University, New Haven, 576pp.

Bigelow, H.B. \& Schroeder, W.C. (1953) Fishes of the Gulf of Maine. Fishery Bulletin, 53 (1), $1-630$.

Bigelow, H.B., Schroeder, W.C. \& Springer, S. (1953) New and little-known sharks from the Atlantic and from the Gulf of Mexico. Bulletin of the Museum of Comparative Zoology at Harvard College, 109 (3), 213-276.

Bilecenoglu, M., Kaya, M., Cihangir, B. \& Çiçek, E. (2014) An updated checklist of the marine fishes of Turkey. Turkish Journal of Zoology, 38, 901-929.

Blainville, H.M.D. de (1816) Prodrome d' une distribution systematique du regne animal. Bull. Sci. Soc. Philom. Paris, 8, 105-124.

Blainville, H.M.D. de (1825) Vertébrës. Class V. Poissons. In: Desmarest, A.G., Blainville, H.M.D. de, Prévost, A., Serville, A. \& Saint-Fargau, L. (Eds.), Faune Française, ou histoire naturelle, générale et particulière, des animaux qui se trouvent en France. Plassan, Paris, 1820-1830. Vols. 13 \& 14, 1-96.

Bloch, M.E. (1785) Naturgeschichte der Ausländischen Fische. Auf Kosten des Verfassers und in Commission bei dem Buchhändler Hr. Hesse, Berlin, 146pp.

Bloch, M.E. (1796) Ichthyologie ou Histoire naturelle des poissons: En six parties avec 216 planches dessinées et enluminées d'après nature. Pt. III. Berlin, pp. 594-1291.

Bocage, J.V.B. \& Capello, F.B.C. (1866) Peixes Plagiostomos. Pt. I: Esqualos. Typographia da Academia Real das Sciencias, Lisboa, 56pp.

Bolaños-Cubillos, N., Abril-Howard, A., Bent-Hooker, H., Caldas, J.P. \& Acero, A. (2015) Lista de peces conocidos del archipiélago de San Andrés y Providencia, Caribe occidental colombiano. Boletín de Investigaciones Marinas y Costeras, 44, 127-162. 
Bonnaterre, P.J. (1788) Tableau encyclopédique et méthodique des trois regnes de la nature. Chez Panckoucke, Paris, 215pp.

Bourne, G.C. (1902) An introduction to the study of the comparative anatomy of animals. Vol. II. The Coelomate Metazoa. George Bell \& Sons, London, 321pp.

Brough, J. (1837) On certain secondary sexual characters in the Commom Dogfish (Scyliorhinus canicula). Proc. Zool. Soc. London (B), 217-273.

Bullis, H.R. \& Thompson, J.R. (1965) Collections of the exploratory fishing vessles Oregon, Silver Bay, Combat and Pelican made during 1956-1960 in the southwestern north Atlantic. SSRF, USFWS 510, 130pp.

Bunkley-Williams, L. \& Williams, E.H. (2004) New locality, depth, and size records and species character modifications of some Caribbean deep-reef/shallow slope fishes and a new host and locality record from the chimaera cestodarian. Caribbean Journal of Science, 40 (1), 88-119.

Burgess, G.H., Link Jr., G.W. \& Ross, S.W. (1979) Additional marine fishes new or rare to Carolina waters. Northeast Gulf Science, 3 (2), 74-87.

Cadenat, J. (1950) Rapport sur les Sélaciens des côtes du Sénégal et plus spécialement sur les Requins. Bulletin de l'Institut Français d'Afrique Noire (A), 12 (4), 944-975.

Cadenat, J. \& Blache, J. (1981) Requins de Méditerranée et d'Atlantique (plus particulièrement de la Côte Occidentale d'Afrique). Fauna Tropicale, 21, 1-330

Calegari, B.B., Delapieve, M.L.S. \& Sousa, L.M. (2014) Tutorial para preparação de mapas de distribuição geográfica. Boletim Sociedade Brasileira de Ictiologia, 118, 15-30.

Canestrini, G. (1872) Fauna d'Italia. Pt. III. Pesci. Fieno (3), Milano, 208pp. 
Capapé, C. (1977) Contribution à la biologie des Scyliorhinidae des côtes tunisiennes. Scyliorhinus canicula (Linné, 1758). Repartition géographique et bathymétrique, sexualité, reproduction, fécondité. Bulletin de l'Office national des Pêches de Tunisie, 1, 83-101.

Capapé, C., Tomasini, J.A. \& Bouchereau, J.-L. (1991) Observations sur la biologie de la reproduction de la petite roussette Scyliorhinus canicula (Linnaeus, 1758) (Pisces, Scyliorhinidae) du Golfe du Lion (France Meridionale). Ichthyophysiologica Acta, 13, 87109.

Capapé, C., Tomasini, J.A. \& Quignard, J.-P. (2000) Les elasmobranches pleurotrêmes de la côte du Languedoc (France Méridionale): observations biologiques et demographiques. Vie et Milieu, 50, 123-133.

Capapé, C., Vergne, Y., Vianet, R., Guélorget, O. \& Quignard, J.-P. (2006) Biological observations on the nursehound, Scyliorhinus stellaris (Linnaeus, 1758) (Chondrichthyes: Scyliorhinidae) in captivity. Acta Adriatica, 47 (1), 29-36.

Capapé, C., Vergne, Y., Reynaud, C., Guélorget, O. \& Quignard, J.-P. (2008) Maturity, fecundity and occurrence of the smallspotted catshark Scyliorhinus canicula (Chondrichthyes: Scyliorhinidae) off the Languedocian coast (southern France, northwestern Mediterranean). Vie et Milieu, 58 (1), 47-55.

Cappetta, H. (2012) Handbook of Paleoichthyology. Vol. 3E: Chondrichthyes. Mesozoic and Cenozoic Elasmobranchii: Teeth. Verlag Dr. Friedrich Pfeil. 512pp.

Cardinale, M. \& Osio, G.C. (2013) Status of Mediterranean and Black Sea resources in European Waters in 2013. Results for stocks in GSA 1-29 (Mediterranean and Black Sea). Presentation at DG MARE, EC, 17 September 2013. http://www.europarl.europa.eu/document/activities/cont/201312/20131217ATT76355/201 31217ATT76355EN.pdf (Acesso em 30 março de 2018).

Carpenter, K.E. \& Niem, V.H. (1998) FAO species identification guide for fishery purposes. The living marine resources of the Western Central Pacific. Vol. 2. Cephalopods, crustaceans, holothurians and sharks. FAO, Rome, pp. 687-1396. 
Carpenter, K.E. \& Angelis, N. de (2016) FAO species identification guide for fishery purposes. The living marine resources of the Eastern Central Atlantic. Vol. 2. Bivalves, gastropods, hagfishes, sharks, batoid fishes and chimaeras. FAO, Rome, 665-1509.

de Carvalho M.R. (1996) Higher-lever elasmobranch phylogeny, basal squaleans, and paraphyly. In: Stiassny, M.L.J., Parenti, L.R. \& Johnson, G.D. (Eds.), Interrelationships of fishes. Academic Press, New York, pp. 35-62.

Castro, J.I, Bubucis, P.M. \& Overstrom, N.A. (1988) The reproductive biology of the chain dogfish, Scyliorhinus retifer. Copeia, 1988 (3), 740-746.

Castro, J.I. (2011) The sharks of North America. Oxford University Press, New York, 640pp.

Cervigón, F. (1960) Peces recogidos en el curso de las campañas realizadas a bordo del Costa Canaria desde Cabo Bojador hasta Guinea Portugesa (África Occidental) y consideraciones sobre su distribución. Invest. Peq. Barcelona, 17, 33-107.

Cervigón, F. (1966) Los peces marinos de Venezuela. Vols. 1 e 2. Monografía, Fundación La Salle, Caracas, 951pp.

Cervigón, F. (1975) Los peces marinos de Venezuela. Complemento IV. Contr. Cient., Univ. Oriente, 5, 1-45.

Cervigón, F. \& Alcalá, A. (1999). Los peces marinos de Venezuela: Tiburones y rayas. Vol. 5. Fundación Museo del Mar, Estado Nueva Esparta, 230pp.

Chu, Y.T. \& Wang, Y.H. (1964). On the geographical distribution and faunal characteristics of the chondrichthian fishes of China. Acta Zoologica, 16, 674-689.

Ciena, A.P., Rangel, B.S., Bruno, C.E.M., Miglino, M.A., de Amorim, A.F., Rici, R.E.G. \& Watanabe, I. (2016) Morphological Aspects of Oral Denticles in the Sharpnose Shark Rhizoprionodon lalandii (Müller \& Henle, 1839) (Elasmobranchii, Carcharhinidae). Anatomia, Histologia, Embryologia, 45 (2), 109-114. 
Collett, R. (1875) Norges fiske, med bemaerkninger om deres Udbredelse. Christiania. Norges fiske, med bemaerkninger om deres Udbredelse, 1-240.

Compagno, L.J.V. (1970) Systematics of the genus Hemitriakis (Selachii: Carcharhinidae), and related genera. Proceedings of the California Academic Sciences, 4 (38), 63-98.

Compagno, L.J.V. (1984) FAO species catalogue. Vol. 4. Sharks of the world: An annotated and illustrated catalogue of shark species known to date. Part 2. Carcharhiniformes. FAO, 4 (125), 251-655.

Compagno, L.J.V. (1988a) Sharks of the Order Carcharhiniformes. Princeton University Press, Princeton, 572pp.

Compagno, L. J. V. (1988b) Scyliorhinus comoroensis sp. n., a new catshark from the Comoro Islands, western Indian Ocean (Carcharhiniformes, Scyliorhinidae). Bull. Mus. Natn. d'Histoire Naturelle, 4, 10 (3), 603-625.

Compagno, L.J.V. (1999) Checklist of living elasmobranchs. In: Hamlett, W.C. (Ed.), Sharks, skates, and rays: the biology of elasmobranch fishes. Johns Hopkins University Press, Maryland, 471-498.

Compagno, L.J.V. (1999) Endoskeleton. In: Hamlett, W.C. (Ed.), Sharks, skates, and rays: The biology of Elasmobranch fish. The Johns Hopkins University Press, Baltimore, 69-92.

Compagno, L.J.V. (2001) Sharks of the world. An annotated and illustrated catalogue of sharks known to date. Volume 2. Bullhead, mackerel and carpet sharks (Heterodontiformes, Lamniformes and Orectolobiformes). FAO species catalogue for fishery purposes. FAO, Rome, 269pp.

Compagno, L.J.V. (2002) Sharks. In: Carpenter, K.E. (Ed.), The living marine resources of the western Central Atlantic. Vol. 1: Introduction, molluscs, crustaceans, hagfishes, sharks, batoid fishes and chimaeras. FAO Species Identification Guide for Fisheries Purposes and American Society of Ichthyologists and Herpetologists Special Publication, 5, 357-505. 
Compagno, L.J.V. (2016) Sharks. In: Carpenter, K.E. \& de Angelis, N. (Eds.), The living Marine Resources of the Eastern Central Atlantic: Bivalves, gastropods, hagfishes, sharks, batoid fishes, and chimaeras. FAO Species Identification Guide for Fisheries Purposes, 1123-1336.

Compagno, L.J.V., Ebert, D.A. \& Cowley, D. (1991) Distribution of offshore demersal cartilaginous fish (Class Chondrichthyes) off the west coast of southern Africa, with notes on their systematics. S. Afr. J. mar. Sci., 11, 43-139.

Compagno, L.J.V. \& Stevens, J.D. (1993) Atelomycterus fasciatus n.sp., a new catshark (Chondrichthyes: Carcharhiniformes: Scyliorhinidae) from tropical Australia. Records of the Australian Museum, 45 (2), 147-169.

Compagno, L.J.V., Dando, M. \& Fowler S. (2005) Sharks of the World. Princeton University Press, Princeton, 368pp.

Compagno, L.J.V., Last, P.R., Stevens, J.D. \& Alava, M.N.R. (2005) Checklist of Philippine Chondrichthyes. CSIRO Marine Laboratories, 243, 1-109.

Couch, J. (1868) A history of the fishes of the British Islands. Vol. 1. Groombridge and Sons 5, London, 208pp.

Coward, T.A. (1910) The vertebrate fauna of Cheshire and Liverpool Bay. Vol. 2. Witherby \& Co. 326 Highh Holborn, London, 210pp.

Crooks, N., Babey, L., Haddon, W.J., Love, A.C. \& Waring, C.P. (2013) Sexual dimorphisms in the dermal denticles of the lesser-spotted catshark, Scyliorhinus canicula (Linnaeus, 1758). PLoS ONE, 8 (10), e76887.

Crooks, N. \& Waring, C.P. (2014) Sexual dimorphisms in the dermal structure of the lesserspotted catshark, Scyliorhinus canicula (Linnaeus, 1758). Acta Zoologica, 94 (3), 331-334. 
Cuvier, G.L.C.F.D (1817) Le regne animal distribue d'apres son organisation, pour servir de base a l'histoire naturelle des animaux et d'introduction a l'anatomie comparée. Paris, 2, 532pp.

Cuvier, G.L.C.F.D. (1829) Le règne animal, distribué d'après son organisation, pour servir de base à l'histoire naturelle des animaux et d'introduction à l'anatomie comparée. Déterville, de l'Impr. de A. Belin, Paris, 2, 584pp.

Cuvier, G.L.C.F.D., Griffith, E. \& Smith, C. (1834) The animal kingdom: arranged in conformity with its organization. The class Pisces. London, 10, 1-680.

Daniel, J.F. (1934) The elasmobranch fishes. 3 ed. University of California Press, Berkeley, 332pp.

Danois, E. (1813) Contribution a l'étude de systématique et biologique poisons de la Manche Occidentale. In: Joubin, L. (Ed.), Annales de l'Institut océanographique. Tome V. Monaco et Cie, Paris, pp. 1-214.

Day, F. (1878) The Fishes of India; being a natural history of the fishes known to inhabit the seas and fresh waters of India, Burma, and Ceylon. B. Quaritch, London, 4, 553-779.

Day, F. (1880-1884) The fishes of Great Britain and Ireland. Williams and Norgate, Edinburgh, 1, 336pp.

de Beer, G.R. (1937) The Development of the Vertebrate Skull. University of Chicago Press, Chicago, 554pp.

Doderlein, P. (1879-1880) Manuale ittiologico del Mediterraneo: ossia sinossi metodica delle varie specie di pesci riscontrate nel Mediterraneo ed in particolare nei mari di Sicilia. Tipografia del Giornale di Sicilia, Palermo, 256pp.

Duhamel du Monceau, H.L. \& La Marre, L.H. (1769-1782) Traite general des peches, et histoire des poissons qu'elles fournissent, etc. Paris, 4 vols. 
Duméril, A.H.A. (1865) Histoire naturelle des poissons ou ichthyologie générale. Tome Premier. Elasmobranchés. Plagiostomes et Holocéphales ou Chimères. Librairie Encyclopédique de Roret, Paris, 720pp.

Duperrey, M.L.I (1830) Voyage autour du monde exécuté par ordre du roi, sur la corvette de Sa Majesté, la Coquille, pendant les années 1822, 1823, 1824, et 1825. Tome II. Arthus Bertrand, Paris, 155pp.

Dyldin, Y.V. (2015) Annotated checklist of the sharks, batoids and chimaeras (Chondrichthyes: Elasmobranchii, Holocephali) from waters of Russia and adjacent areas. Publications of the Seto Marine Biological Laboratory, 43, 40-91.

Ebert, D.A., Compagno, L.J.V. \& Cowley, P.D. (2006) Reproductive biology of catsharks (Chondichthyes: Scyliorhinidae) off the west coast of southern Africa. ICES Journal of Marine Science, 63, 1053-1065.

Ebert, D.A., Fowler, S., Compagno, L.J.V. \& Dando, M. (2013) Sharks of the World: A Fully Illustrade Guide. Wild Nature Press, Plymouth, 528pp.

Ebert, D.A. \& Mostarda, E. (2013) Identification guide to the deep-sea cartilaginous fishes of the Indian Ocean. FishFinder Programme, FAO, Rome, 76pp.

Ebert, D.A. \& Stehmann, M. (2013) Sharks, batoids, and chimaeras of the North Atlantic. FAO Species Catalogue for Fishery Purposes. FAO, Rome, 7, 523pp.

Ellis, J. R. \& Shackley, S. E. (1995) Ontogenic changes and sexual dimorphism in the head, mouth and teeth of the lesser spotted dogfish. Journal of Fish Biology, 47, 155-164.

Ellis, J.R. \& Shackley, S.E. (1997) The reproductive biology of Scyliorhinus canicula in the Bristol Channel, UK. Journal of Fish Biology, 51 (2), 361-372.

Ellis, J.R., Cruz-Martinez, A., Rackham, B.D. \& Rogers, S.I. (2005) The distribution of chondrichthyan fishes around the British Isles and implications for conservation. $J$. Northwest Atl. Fish Sci., 35, 195-213. 
Erdogan, Z.A., Koç, H.T., Cakir, D.T., Nerlovic, V. \& Dulcic, J. (2004) Sexual dimorphism in the small-spotted catshark, Scyliorhinus canicula (L., 1758), from the Edremit Bay (Turkey). Annales, Series Historia Naturalis, 14 (2), 165-170.

Espinosa Pérez, H. \& Huidobro Campos, L. (1995) Nuevos registros de tiburones en México. Revista de la Sociedad Mexicana de Historia Natural, 46, 113-120.

Faber, G.L. (1883) The fisheries of the Adriatic and the fish thereof: a report of the AustroHungarian sea-fisheries, with a detailed description of the marine fauna of the Adriatic Gulf. Bernard Quaritch, London, 292pp.

Farris, J. (1969) A successive approximations approach to character weighting. Systematic Zoology, 18 (4), 374-385.

Feng, D. \& Knight, D.P. (1994) Structure and Formation of the Egg Capsule Tendrils in the Dogfish Scyliorhinus canicula. Philosophical Transactions of the Royal Society of London, Series B, Biological Sciences, 343 (1305), 285-302.

Figueiredo, J. L. (1977) Manual de Peixes Marinhos do Sudeste do Brasil. I. Introdução. Cações, Raias e Quimeras. Museu de Zoologia da Universidade de São Paulo, São Paulo, $104 \mathrm{pp}$.

Filiz, H \& Taskavak, E. (2006) Sexual dimorphism in the head, mouth, and body morphology of the smallspotted catshark, Scyliorhinus canicula (Linnaeus, 1758) (Chondrichthyes: Scyliorhinidae) from Turkey. Acta Adriatica, 47 (1), 37-47.

Fischer, W. \& Bianchi, G. (1984) FAO Species Identification Sheets for Fishery Purposes. Western Indian Ocean (Fishing Area 51). Prepared and Printed with the Support of the Danish International Development Agency (DANIDA). FAO, Rome, Vols. 1-6.

Fischer, W., Bauchot, M.L. \& Schneider, M.R. (1987) Fiches FAO d'identification des espèces pour les besoins de la pêche. (Révision 1). Méditerranée et Mer Noire. Zone de pêche 37. FAO. Rome, 2, 761-1530. 
Flammang, B.E., Ebert, D.A. \& Cailliet, G.M. (2008) Reproductive biology of deep-sea catsharks (Chondrichthyes: Scyliorhinidae) in the eastern North Pacific. Environmental Biology of Fishes, 81 (1), 35-49.

Flammang, B.E., Ebert, D.A. \& Caillet, G.M. (2007) Egg cases of the genus Apristurus (Chondrichthyes: Scyliorhinidae): Phylogenetic and ecological implications. Zoology, 110 (4), 308-317.

Fleming, J. (1828) A history of British animals: exhibiting the descriptive characters and systematical arrangement of the genera and species of quadrupeds, birds, reptiles, fishes, mollusca, and radiata of the United Kingdom; including the indigenous, extirpated, and extinct kinds, together with periodical and occasional visitants. Bell \& Bradfute, Edinburgh, $565 \mathrm{pp}$.

Ford, E. (1921) A contribution to our knowledge of the life-histories of the dogfishes landed at Plymouth. J. Mar. Biol. Assoc. U.K., 12, 468-505.

Fowler, H.W. (1934) Descriptions of new fishes obtained 1907 to 1910, chiefly in the Philippine Islands and adjacent seas. Proc. Acad. Nat. Sci. Phila. 85, 233-367.

Fowler, H.W. (1941) The fishes of the groups Elasmobranchi, Holocephali, Isospondyli, and Ostariophysi obtained by the United States Bureau of Fisheries steamer "Albattross" in 1907 to 1910, chiefly in the Philippine Islands and adjacent seas. Bulletin U. S. National Museum, 13, 879pp.

Fricke, R. (1999) Annotated checklist of the marine and estuarine fishes of Germany, with remarks on their taxonomic identity. Stuttgarter Beiträge zur Naturkunde (A), 587, 1-67.

Fricke, R., Bilecenoglu, M. \& Sari, H.M. (2007) Annotated checklist of fish and lamprey species (Gnathostomata and Petromyzontomorphi) of Turkey, including a Red List of threatened and declining species. Stuttgarter Beiträge zur Naturkunde (A), 706, 1-169. 
Fricke, R., Eschmeyer, W. N. \& van der Laan, R. (eds) 2018. Catalog of fishes: genera, species, references. (http://researcharchive.calacademy.org/research/ichthyology/ catalog/fishcatmain.asp). Acessado em 13 de setembro de 018.

Gadig, O. B. F. (2001) Tubarões da costa brasileira. 2001. 343f. (Tese de Doutorado) Universidade Estadual Paulista, Instituto de Biociências de Rio Claro, Rio Claro, SP.

Gadig, O. B. F. \& Gomes, U. L. (2003) Classe Chondrichthyes. In: Menezes, N. A., Buckup, P. A., Figueiredo, J. L. \& Moura, R. L. (Eds.), Catálogo das espécies de peixes marinhos do Brasil. Museu de Zoologia da Universidade de São Paulo, São Paulo, pp. 21-22.

Garman S. (1881) New species of selachians in the museum collection. Bull. Mus. Comp. Zool., $6,167-172$.

Garman, S. (1913) The Plagiostomia (Sharks, skates and rays). Memoirs of the Museum of Comparative Zoology at Harvard College, 36, 515pp.

George, M.R. (2009) An annotated checklist of North Sea cartilaginous fish species. Journal of Applied Ichthyology, 25 (S1), 33-39.

Gesner, C. (1586) Conr. Gesneri Tigurini medicinae et philosophiae professoris in Schola Tigurina, Historia animalium liber II: qui est de quadrupedibus ouiparis. Francofurd Eofficina typographica Ioannis Wecheli, Impensis Roberti Cambieri, 806pp.

Gibson, C., Valenti, S.V, Fowler, S.L. \& Fordham, S.V. (2006) The Conservation Status of Northeast Atlantic Chondrichthyans. IUCN Species Survival Commission, Shark Specialist Group, Newbury, Reino Unido, 76pp.

Gilchrist, J. D. F. (1902) Catalogue of fishes recorded from South Africa. Mar. Invest. S. Afr., $1,97-179$.

Gilchrist, J. D. F. (1921) Fisheries and Marine Biological Survey. Report no. 1 for the Year 1920. Rep. Fish. mar. Biol. Surv. Un. S. Vols. 1-4. 
Gill, T. N. (1862). Analytical synopsis of the order of Squali; and revision of the nomenclature of the genera. Ann. Lye. Nat. Hist., 7, 367-408.

Gohar, H.A.F. \& Mazhar, F.M. (1964) Elasmobranchs of the northwestern Red Sea. Publ. Mar. Biol. Stn. Al-Ghardaqa, 13, 1-144.

Goloboff, P.A. (1993) Estimating character weights during tree search. Cladistics, 9, 83-91.

Goloboff, P.A. (1995) Parsimony and weighting: a reply to Turner and Zandee. Cladistics, 11, 91-104.

Goloboff, P., Farris, J. \& Nixon, K.C. (2003) T.N.T. Tree Analysis Using New Technology. Disponível em - http://www.zmuc.dk/public/phylogeny/tnt.

Goloboff, P.A., Mattoni, C.M. \& Quinteros, A.S. (2006) Continuous characters analyzed as such. Cladistics, 22, 589-601.

Goloboff, P.A., Carpenter, J.M., Arias, J.S. \& Esquivel, D.R.M. (2008) Weighting against homoplasy improves phylogenetic analysis of morphological data sets. Cladistics, 24, 1-16.

Goloboff, P.A., Farris, J.S. \& Nixon, K.C. (2008) TNT, a free program for phylogenetic analysis. Cladistics, 24, 774-786.

Gomes, U.L. \& Tomás, A.R.G. (1991) Dimorfismo sexual secundário no cação Scyliorhinus haeckelli Ribeiro, 1907 (Elasmobranchii, Scyliorhinidae). Anais da Academia Brasileira de Ciências, 63 (2), 193-200.

Gomes, U.L. \& de Carvalho, M.R. (1995) Egg Capsules of Schroederichthys tenuis and Scyliorhinus haeckelii (Chondrichthyes, Scyliorhinidae). Copeia, 1995 (1), 232-236.

Gomes, U.L., Peters, G.O., Carvalho, M.R. \& Gadig, O.B.F. (2006) Anatomical investigation of the slender catshark Schroederichthys tenuis Springer, 1966, with notes on intrageneric relationships (Chondrichthyes: Carcharhiniformes: Scyliorhinidae). Zootaxa 1119, 29-58. 
Gomes, U.L., Signori, C.N., Gadig, O.B.F. \& Santos, H.R.S. (2010) Guia para Identificação de Tubarões e Raias do Rio de Janeiro. Technical Books, Rio de Janeiro, 234pp.

Goode, S.B. \& Bean, T.H. (1896) Oceanic ichthyology. Smithson. Inst. Spec. Bull., 533pp.

Gordon, W.J. (1902) Our country's fishes and how to know them: a guide to all the fishes of Great Britain. Simpkin, Hamilton, Kent \& Co, London, 152pp.

Gordon, C.A., Hood, A.R. \& Ellis, J.R. (2016) Descriptions and revised key to the eggcases of the skates (Rajiformes: Rajidae) and catsharks (Carcharhiniformes: Scyliorhinidae) of the British Isles. Zootaxa, 4150 (3), 255-280.

Gosse, P.H. (1851) Natural history: Fishes. Society for promoting Christian knowledge, London, 327pp.

Goto, T. (2001) Comparative Anatomy, Phylogeny and Cladistic Classification of the order Orectolobiformes (Chondrichthyes, Elasmobranchii). Memoirs of the Graduate School of Fisheries Sciences Hokkaido University, 48, 1-100

Gratzianov, V.J. (1906) Über eine besondere Gruppe der Rochen. Zoologischer Anzeiger, 30 (14), 399-406.

Gronovius, L.T. (1756) Bibliotheca regni animalis atque lapidei, seu, Recensio auctorum et librorum: qui de regno animali \& lapideo methodice, physice, medice, chymice, philologice, vel theologice tractant, in usum naturalis historiae studiosorum. Lugduni Batavorum, Sumptibus auctoris, 326pp.

Guinot, G. Adnet, S., Shimada, K., Underwood, C.J., Siversson, M., Wards, D.J., Kriwet, J. \& Cappetta, H. (2018) On the need of providing tooth morphology in descriptions of extant elasmobranch species. Zootaxa 4461 (1), 118-126

Günther, A. (1870) Catalogue of the Fishes in the British Museum. Vol. 8. Taylor \& Francis, London, 549pp. 
Gutiérrez, E.R., Orozco, M.V., Cortés, R., Blanco, A., Alfonso, Y., Delgado, D., Lemus, E. \& Álvarez, I.L. (2015) Catálogo ilustrado de los especímenes tipo de tiburones cubanos. Novitates Caribaea, 8, 40-49.

Hacohen-Domené, A., Polanco-Vásquez, F. \& Graham, R.T. (2016) First report of the whitesaddled catshark Scyliorhinus hesperius (Springer 1966) in Guatemala's Caribbean Sea. Marine Biodiversity Records 9 (1), 101.

Hamilton, R. (1854) Ichthyology. Vol 3. British Fishes, Pt 2. In: Jardine, W. (Ed.), The naturalist's library.Vol. 37. W.H. Lizars, Edinburgh, 424pp.

Hartel, K. E. \& Dingerkus, G. (1997) Types of Garman chondrichthian species in the Museum of Comparative Zoology. In: Reprint of "The Plagiostomia (Sharks, Skates and Rays)" by Samuel Garman. Benthic Press, Los Angeles, pp. xxxvi-xlix.

Henderson, A.C. \& Casey, A. (2001) Reproduction and Size in the lesser-spotted dogfish Scyliorhinus canicula (Elasmobranchii; Scyliorhinidae) from the west coast of Ireland. Cahiers de Biologie Marine, 42, 397-405.

Hennig, W. (1950) Grundzüge einer Theorie der phylogenetischen Systematik. Deutscher Centralverlag, Berlin, 370pp.

Hennig, W. (1965) Phylogenetic systematics. Annu. Rev. Entomol. 10, 97-116.

Heppe, J.C. (1787) Abbildung und Beschreibung der Fische. Samuel Winterschmid, Nürnberg, 306pp.

Herman, J., Hovestad-Euler, M. \& Hovestad, D.C. (1990) Contributions to the study of the comparative morphology of teeth and other relevant ichthyodorulites in living superspecific taxa of Chondrichthyan fishes. Part A: Selachii. No. 2b: Order: Carcharhiniformes Familiy: Scyliorhinidae. Bulletin de l'Institut Royal des Sciences Naturelles de Belgique, Biologie, 60, 181-230. 
Herre, A.W.C.T. (1953) Check list of Philippine fishes. Fisheries Wildlife Service, Ru. Rep., 20, 1-977.

Hilgendorf, F.M. (1904) Ein neuer Scyllium-artiger Haifisch, Proscyllium habereri nov. subgen. n. spec. von Formosa. Sitzungsberichte der Gesellschaft naturforschender Freunde zu Berlin, 1904 (2), 39-41.

Hoffman, H.A. \& Jordan, D.S. (1892) A catalogue of the fishes of Greece with notes on the names now in use and those employed by classical authors. Academy of Natural Sciences of Philadelphia, 44, 230-286.

Honma, Y. (1952) A list of the fishes collected in the Province of Echigo, including

Sado Island. Japanese Journal of Ichthyology, 2, 138-146.

Houttuin, F. (1764) Natuurlyke historie: of, Uitvoerige beschryving der dieren, planten, en mineraalen. Amsterdam, 500pp.

Howell-Rivero, L. (1936) Some new, rare and little-known fishes from Cuba. Proc. Boston Soc. Nat. Hist., 41, 41-76.

Huber, D.R., Soares, M.C. \& de Carvalho, M.R. (2011) Cartilaginous Fishes Cranial Muscles. In: Farrell, A.P. (Ed.), Encyclopedia of Fish Physiology: From Genome to Environment. Academic Press, San Diego, 1, 449-462.

Human, B.A., Owen, E.P., Compagno, L.J.V. \& Harley, E.H. (2006) Testing morphologically based phylogenetic theories within the cartilaginous fishes with molecular data, with special reference to the catshark family (Chondrichthyes; Scyliorhinidae) and the interrelationships within them. Molecular Phylogenetics and Evolution, 39 (2006), 384-391.

Iglésias, S.P., Lecointre, G. \& Sellos, D.Y. (2005) Extensive paraphylies within sharks of the order Carcharhiniformes inferred from nuclear and mitochondrial genes. Molecular Phylogenetics and Evolution, 34 (2005), 569-583. 
International Commission on Zoological Nomenclature (1999) International Code of Zoological Nomenclature, Fourth Edition: adopted by the International Union of Biological Sciences. Disponível em: http://iczn.org/code.

Ivory, P.F., Jeal, F. \& Nolan, C.P. (2004) Age Determination, Growth and Reproduction in the Lesser-spotted Dogfish, Scyliorhinus canicula (L.). Journal of Northwest Atlantic Fishery Science, 35, 89-106.

Jenyns, L. (1835) A manual of British vertebrate animals: or Descriptions of all the animals belonging to the classes, Mammalia, Aves, Reptilia, Amphibia, and Pisces, which have been hitherto observed in the British Islands: including the domesticated, naturalized, and extirpated species: the whole systematically arranged. J. \& J.J. Deighton, Cambridge, 559pp.

Jordan, D.S. \& Evermann, B.W. (1896) The fishes of North and Middle America. Bull. U.S. Natl. Mus., 47, 1-1240.

Jordan, D.S. \& Gilbert, C.H. (1882) Synopsis of fishes of North America. Bulletin United States National Museum, 16, 1018pp.

Jordan, D.S., Tanaka, S. \& Snyder, J.O. (1913) A catalogue of the fishes of Japan.

J. Coll. Sci., Tokyo Imp. Univ., 33, 1-497.

Jungersen, H. F. E. (1899) On the apendices genitales in the greenland shark Somniosus microcephalus (Bl. Schn.) and another selachians. Danish Ingolf Expedition. Vol. II. Bianco luno, Copenhagen, 88pp.

Kamohara, T. (1950) Description of tke Fishes from tke Province of Tosa and Kisku, Japan. Kochi Ken Bunkyo Kyokai, Kochi, 288pp.

Kerr, J.G. (1921) Zoology for medical students. Macmilan \& Co., London, 477pp. 
Kimber, J.A., Sims, D.W., Bellamy, P.H. \& Gill, A.B. (2009) Male-female interactions affect foraging behaviour within groups of small-spotted catshark, Scyliorhinus canicula. Animal Behaviour, 77 (6), 1435-1440.

Kiraly, S.J., Moore, J.A. \& Jasinsky, P.H. (2003) Deepwater and Other Sharks of the U.S. Atlantic Ocean Exclusive Economic Zone. Marine Fisheries Review, 65 (4), 1-63.

Klein, J.T. (1742) Historiae piscium naturalis promovendae missus tertus de piscibus per branchias ocultas spirantibus au justum numerim et ordinem redigendis. Gedani, Litteris Schriberianis, 46pp.

Kousteni, V., Kasapidis, P., Kotoulas, G. \& Megalofonou, P. (2014) Strong population genetic structure and contrasting demographic histories for the small-spotted catshark (Scyliorhinus canicula) in the Mediterranean Sea. Journal of Heredity, 114 (3), 333-343.

Kyne, P.M., Carlson, J.K., Ebert, D.A., Fordham, S.V., Bizzarro, J.J., Graham, R.T., Kulka, D.W., Tewes, E.E., Harrison, L.R. \& Dulvy, N.K. (2012) The Conservation Status of North American, Central American, and Caribbean Chondrichthyans. IUCN Species Survival Commission Shark Specialist Group, Vancouver, Canada.

La Cépède, B.G.E.L.V.I. (1830) Oeuvres du comte de Lacépède: comprenant l'histoire naturelle des quadrupèdes ovipares, des serpents, des poissons et des cétacés. Paris, 399pp.

Lasso, C.A., Lasso-Alcalá, O.M. \& Capelo, C.J. (1998) Catálogo de la colección de tipos de peces de la Fundación La Salle de Ciencias naturales. Parte I: Museo de Historia Natural la Salle (MHNLS). Memoria, Fundación La Salle de Ciencias Naturales, 57 (147), 37-52.

Last, P.R., Séret, B. \& White, W.L. (2008) New swellsharks (Cephaloscyllium: Scyliorhinidae) from the Indo-Australian region. CSIRO Marine and Atmospheric Research Paper, 22, $129-146$.

Last, P.R. \& White, W.L. (2008) Two new saddled swellsharks (Cephaloscyllium: Scyliorhinidae) from eastern Australia. CSIRO Marine and Atmospheric Research Paper, 22, 159-170. 
Leible, M., Dittus, D.M. \& Belmar, C.G.G. (1982) Atlas Anatomico de Pintarroja Schroederichthys chilensis (Guichenot, 1848) (Chondrichthyes: Scyliorhinidae). Vol. II. Sistemas: Muscular, Esqueletico, Respiratorio y Digestivo. Pontificia Universidad Catolica de Chile, Talcahuano, 79pp.

Leigh-Sharpe, W.H. (1920) The comparative morphology of the secondary sexual characters of elasmobranch fishes. The claspers, clasper siphons, and clasper glands. Memoir I. Journal of Morphology and Physiology, 34 (2), 245-265.

Leigh-Sharpe, W.H. (1921) The comparative morphology of the secondary sexual characters of elasmobranch fishes. The claspers, clasper siphons, and clasper glands. Memoir II. Journal of Morphology and Physiology, 35, 2, 359-380.

Leigh-Sharpe, W.H. (1922a) The comparative morphology of the secondary sexual characters of elasmobranch fishes. The claspers, clasper siphons, and clasper glands. Memoir III. Journal of Morphology and Physiology, 36 (2), 191-198.

Leigh-Sharpe, W.H. (1922b) The comparative morphology of the secondary sexual characters of holocephali and elasmobranch fishes. The claspers, clasper siphons, and clasper glands. Memoir V. Journal of Morphology and Physiology, 36 (2), 221-243.

Leigh-Sharpe, W.H. (1924a) The comparative morphology of the secondary sexual characters of elasmobranch fishes. The claspers, clasper siphons, and clasper glands. Memoir VI. Journal of Morphology and Physiology, 39 (2), 553-566.

Leigh-Sharpe, W.H. (1924b) The comparative morphology of the secondary sexual characters of elasmobranch fishes. The claspers, clasper siphons, and clasper glands. Memoir VII. Journal of Morphology and Physiology, 39 (2), 567-577.

Leigh-Sharpe, W.H. (1926a) The comparative morphology of the secondary sexual characters of elasmobranch fishes. The claspers, clasper siphons, and clasper glands. Memoir VIII. Journal of Morphology and Physiology, 42 (1), 307-320. 
Leigh-Sharpe, W.H. (1926b) The comparative morphology of the secondary sexual characters of elasmobranch fishes. The claspers, clasper siphons, and clasper glands. Memoir IX. Journal of Morphology and Physiology, 42 (1), 321-334.

Leigh-Sharpe, W.H. (1926c) The comparative morphology of the secondary sexual characters of elasmobranch fishes. The claspers, clasper siphons, and clasper glands. Memoir X. Journal of Morphology and Physiology, 42 (1), 335-348.

Leloup, J. \& Olivereau, M. (1951) Donnees biometriques comparatives sur la roussette (Scyllium canicula L.) de la Manche et de la Mediterranee. Vie et Milieu, 2, 182-209.

Lindberg, G.U. \& Legeza, M.1. (1967) Fishes of the Sea of Japan and the Adjacent Areas of the Sea of Okhotsk and the Yellow Sea. Part 1. Israel Program for Sci. Transl., Jerusalem, 198pp.

Linnaeus, C. (1758) Systema Naturae, ed. X, 824pp.

Linnaeus, C. (1766) Systema Naturae, ed. XII, 532pp.

Litivinov, F.F. (2003) Sexual dimorphism as an index of the isolation of West African populations of the cat shark Scyliorhinus canicula. Journal of Ichthyology 43 (1), 81-85.

Lozano Rey, L. (1928) Fauna Iberica. Peces. Mus. Nac. Ciencas Nat. Madrid, 1, 1-692.

Lyle, J.M. (1983) Food and feeding habits of the lesser spotted dogfish, Scyliorhinus canicula (L.), in Isle of Man waters. Journal of Fish Biology, 23 (6), 725-737.

Lynghammar, A., Christiansen, J.S., Mecklenburg, C.W., Karamushko, O.V., Møller, P.R. \& Gallucci, V.F. (2013) Species richness and distribution of chondrichthyan fishes in the Arctic Ocean and adjacent seas. Biodiversity, 14 (1), 57-66.

Maisey, J.G. (1984) Higher elasmobranch phylogeny: a look at the evidence. J. Vert. Paleontol., 4 (3), 359-371. 
Marinelli, W. \& Strenger, A. (1959) Vergleichende Anatomie und Morphologie der Wirbeltiere III Lieferung (Squalus acanthias). Franz Deutick, Vienna.

Matsubara, K. (1936) Order Plagiostomi I. Fauna Nipponica, 15, 1-160.

Matsubara, K. (1955). Fish Morphology and Hierarchy. Vols. I-III. Ishizaki Shoten, Tokyo, $1605 \mathrm{pp}$.

Maurin, C. \& Bonnet, M. (1970) Poissons des cotes nord-ouest Africaines (Campagnes de la "Thalassa" 1962 et 1968). Rev. Trav. Inst. Peches Marit., 34, 125-169.

McLaughlin, D.M. \& Morrissey, J.F. (2004) New records of elasmobranchs from the Cayman Trench, Jamaica. Bulletin of Marine Science, 75 (3), 481-485.

Mejía-Falla, P.A., Navia, A.F., Mejía-Ladino, L.M., Acero, A.P. \& Rubio, E.A. (2007) Tiburones y rayas de Colombia (Pisces: Elasmobranchii). Lista revisada, actualizada y comentada. Boletín de Investigaciones Marinas y Costeras, 34, 128-169.

Menni, R.C., Ringuelet, R.A. \& Aramburu, R.H. (1984) Peces marinos de la Argentina y Uruguay. Editorial Hemisferio Sur S.A, Buenos, Aires, Argentina, 359pp.

Metten, H. (1939) Studies on the reproduction of the dogfish. Philosophical Transactions of the Royal Society of London, 230, 217-238.

Miranda Ribeiro, A. M. (1907) Fauna brasiliense. Peixes. II (Desmobranchios). Archivos Museu Nacional 14, 2-20.

Moore, J.A., Hartel, K.E., Craddock, J.E. \& Galbraith, J.K. (2003) An annotated list of deepwater fishes from off the New England region, with new area records. Northeastern Naturalist, 10 (2), 159-248.

Moreau, E. (1881) Histoire naturelle des poissons de la France. Tome I. Paris, 480pp.

Mori, T. (1952) Check list of the fishes of Korea. Mem. Hyogo Univ. Agr., 1, 1-228. 
Mori, T. (1956) Fishes of San-in District including Oki Islands and its adjacent waters (Southern Japan Sea). Mem. Hyogo Univ. Agr, 2, 1-62.

Moss, S.A. (1972) The feeding mechanism of sharks of the family Carcharhinidae. J. Zool. London, 167, 423-436.

Motta, P.J. \& Wilga, C.D. (1995) Anatomy of the feeding apparatus of the lemon shark, Negaprion brevirostris. Journal of Morphology, 226 (3), 309-329.

Müller, J. \& Henle, J. (1838-41). Systematische Beschreibung der Plagiostomen. Viet und Comp., Berlin, 200pp.

Muñoz Chápuli, R. (1985) Sobre la clasificación tipológica del esqueleto dérmico de escualos (Chondrichthyes). Miscellània Zoològica, 17, 283-285.

Muñoz-Chápuli, R. \& Ruiz, M.B. (1984) Tendencias generales del crecimiento relativo em escualos. Investigacion Pesquera, 48 (2), 303-317.

Nakaya, K. (1975) Taxonomy, comparative anatomy and phylogeny of Japanese catsharks, Scyliorhinidae. Memoirs of the Faculty of Fisheries, Hokkaido University, 23, 1-94.

Nakaya, K. \& Shirai, S. (1992) Fauna and Zoogeography of Deep-Benthic chondrichthyan Fishes around the Japanese Archipelago. Japanese Journal of Ichthyology, 39 (1), 37-48.

Nakaya, K., Inoue, S. \& Ho, H.-C. (2013) A review of the genus Cephaloscyllium (Chondrichthyes: Carcharhiniformes: Scyliorhinidae) from Taiwanese waters. Zootaxa, $3752,101-129$.

Naylor, G. J. P., Caira, J.N., Jensen, K., Rosana, K.A.M., Straube, N. \& Lakner, C. (2012) Elasmobranch phylogeny: A mitochondrial estimate based on 595 species. In: Carrier, J.C., Musick, J.A. \& Heithaus, M.R. (Eds.), Biology of Sharks and Their Relatives (2nd ed.). CRC Press, Boca Raton, pp. 31-56. 
Nelson, G.J. (1970) Pharyngeal Denticles (Placoid Scales) of Sharks, with Notes on the Dermal Skeleton of Vertebrates. American Museum Novitates, 2415, 1-53.

Nelson. G.J. \& Platnick, N.I. (1981) Systematics and biogeography: cladistics and vicariance. Columbia University Press, 567pp.

Nelson, J.S., Grande, T.C. \& Wilson, M.V.H. (2016) Fishes of the World. $5^{\mathrm{a}}$ ed. John Wiley \& Sons, New Jersey, 707pp.

Nunan, G.W. \& Senna, M. L.V. (2007) Tubarões (Selachii) coletados pelo navio oceanográfico Thalassa sobre a plataforma externa e talude continental do Brasil entre $11^{\circ}$ e $22^{\circ} \mathrm{S}$. In: Costa, P. A. S., Olavo, G. \& Martins, A.S. (Eds.), Biodiversidade da fauna marinha profunda na costa central brasileira. Museu Nacional, Rio de Janeiro, 163-183.

O’Donoghue, C.H. (1921) An introduction to zoology. Appleton and Co, New York, 501pp.

Parker, T.J. \& Haswell, W.A. (1897) A text-book of zoology. Ed. 1, vol. 2 (chordates). Macmillan, London, 680pp.

Parker, T.J. (1914) Lessons in elementary biology. Macmillan, London,

Parnell, R. (1838) Prize essay on the natural and economical history of the fishes marine, fluviatile, and lacustrine, of the river district of the Firth of Forth. Neill \& Co Parsons, Edinburgh, pp. 161-520.

Partington, C.F. (1837) The British cyclopadia of natural history: combining a scientific classification of animals, plants, and minerals. W. S. Orr \& Co., Amen Corner, Paternosterrow, London, 880pp.

Pennant, T. (1812) British zoology. Vol. 3. London, 546pp.

Piestchmann, V. (1906) Ichthyologische Ergebnisse einer Reise nach Island, an die atlantische Küste von Marokko und in die westliche Hälfte des Mittelmeeres. Annalen des Naturhistorischen Museums in Wien, 21, 72-148. 
Pietschmann, V. (1908). Japanische Plagiostomen. Sitzungsber. Kais., Akad. Wiss. Wien, 117, $637-710$.

Poll, M. (1951) Poissons, I. Generalites. II. Selaciens et Chimeres. Inst. R. Sci. Nat. Belg., Exped. Oceanogr. Belg. Eaux Cot. Afr. Atlant. Sud, 4, 1-154.

Randall, J.E. \& Lim, K.K.P. (2000) A checklist of the fishes of the South China Sea. Raffles Bulletin of Zoology, Supplement 8, 569-667.

Rangel, B.S., Wosnick, N., Hammerschlag, N., Ciena, A.P., Kfoury, J.R. \& Rici, R.E.G. (2017) A preliminary investigation into the morphology of oral papillae and denticles of blue sharks (Prionace glauca) with inferences about its functional significance across life stages. Journal of Anatomy, 203 (3), 389-397.

Regan, C.T. (1908). A synopsis of the sharks of the family Scyliorhinidae. Ann. Mag. Nat. Hist., 8, 1, 453-465.

Reif, W. E. (1982) Morphogenesis and function of the squamation in sharks. 1. Comparative functional morphology of shark scales, and ecology of sharks. Neues Jahr. Geol. Paläontol. $164,172-183$.

Reif, W.E. (1985) Squamation and ecology of sharks. Cour. Forsch. Inst. Senckenberg, 78, 1101.

Rey, J. Sola, L.G.\& Massutí, E. (2004) Distribution and biology of the blackmouth catshark Galeus melastomus in the Alboran Sea (Southwestern Mediterranean). J. Northw. Atl. Fish. Sci., 35, 215-223

Rincon, G., Mazzoleni, R.C., Palmeira, A.R.O. \& Lessa, R. (2017) Deep-Water Sharks, Rays, and Chimaeras of Brazil. In: Rodrigues Filho, L.F.S. (Ed.), Chondrichthyes Multidisciplinary Approach Edition, Chapter 5. Disponível em: https://www.intechopen.com/books/chondrichthyes-multidisciplinary-approach/deepwater-sharks-rays-and-chimaeras-of-brazil. 
Risso, A. (1826) Histoire naturelle des principales productions de l'Europe méridionale et particulièrement de celles des environs de Nice et des Alpes Maritimes. F.-G. Levrault Libraire, Paris, 402pp.

Rodríguez-Cabello, C., de la Gandara, F. \& Sánchez, F. (1997) Preliminary results on growth and movements of dogfish Scyliorhinus canicula (L., 1758) in the Cantabrian Sea. Oceanol. Acta, 21 (2), 363-370.

Rodríguez-Cabello, C., Velasco, F. \& Olaso, I. (1998) Reproductive biology of lesser spotted dogfish Scyliorhinus canicula (L., 1758) in the Cantabrian Sea. Scientia marina, 62 (3), 187-191.

Rodríguez-Cabello, C., Sánchez, F., Fernández, A. \& Olaso, I. (2004) Is the lesser spotted dogfish (Scyliorhinus canicula) population from the Cantabrian Sea a unique stock? Fisheries Research, 69 (1), 57-71.

Rodríguez-Cabello, C., Sánchez, F. \& Olaso, I. (2007) Distribution patterns and sexual segregations of Scyliorhinus canicula (L.) in the Cantabrian Sea. Journal of Fish Biology, 70 (5), 1568-1586.

Rondelet, G. (1554) Libri de piscibus marinis in quibus verae piscium effigies expressae sunt. Mathias Bonhomme, Lyon.

Sabata, E. de \& \& Cló, S. (2013) Some breeding sites of the nursehound (Scyliorhinus stellaris) (Chondrichthyes, Scyliorhinidae) in Italian waters, as reported by divers. Biol. Mar. Mediterr., 20 (1), 178-179.

Salviani, H. (1554) Aquatilium animalium historiae, liber primus: cum eorumdem formis, aere excusis. Roma, 256pp.

Schaak-da Silva, J.A. \& Ebert, D.A. (2008) A revision of the western North Pacific swellsharks, genus Cephaloscyllium Gill 1862 (Chondrichthys: Carcharhiniformes: Scyliorhinidae), including descriptions of two new species. Zootaxa, 1872, 1-8. 
Schinz, H.R. (1840) Europäische Fauna, oder, Verzeichniss der wirbelthiere Europa's. E. Schweizerbarts Verlagshandlung, Stuttgart, 448pp.

Schmidt, P.J. (1930) A Selachian clasper with a hundred hooks. Copeia, 1930, 48-50.

Serena, F. (2005) Field identification guide to the sharks and rays of the Mediterranean and Black Sea. FAO Species Identification Guide for Fishery Purposes, 97pp.

Séret, B. \& Last, P.R. (2007) Four new species of deep-water catsharks of the genus Parmaturus (Carcharhiniformes: Scyliorhinidae) from New Caledonia, Indonesia and Australia. Zootaxa, 1657, 23-39.

Shirai, S. (1992a) Squalean Phylogeny: a new framework of 'Squaloid' sharks and related taxa. Hokkaido University Press, Sapporo, 151pp.

Shirai, S. (1992b) Identity of extra branchial arches of Hexanchiformes (Pisces, Elasmobranchii). Bulletin of the Faculty of Fishery of the Hokkaido University, 43 (1), 24 32.

Shirai, S. (1996) Phylogenetic interrelationships of Neoselachians (Chondrichthyes: Euselachii). In: Stiassny, M.L.J., Parenti, L.R. \& Johnson, G.D. (Eds.), Interrelationships of fishes. Academic Press, New York, 9-34.

Shirai, S., Hagiwara, S. \& Nakaya, K. (1992) Scyliorhinus tokubee sp. nov. from Izu Peninsula, Southern Japan (Scyliorhinidae, Elasmobranchii). Japanese Journal of Ichthyology 39 (1), 9-16.

Silva, J.P.C.B. \& de Carvalho, M.R. (2015) Morphology and phylogenetic significance of the pectoral articular region in elasmobranchs. Zoological Journal of the Linnean Society, 2015, $1-44$. 
Sims, D.W., Nash, J.P. \& Morritt, D. (2001) Movements and activity of male and female dogfish in a tidal sea lough: alternative behavioural strategies and apparent sexual segregation. Marine Biology, 139 (6), 1165-1175.

Sinohara, G., Nakae, M., Ueda, Y., Kojima, S. \& Matsuura, K. (2014) Annotated checklist of deep-sea fishes of the Sea of Japan. In: Fujita, T. (Ed.), Deep-sea Fauna of the Sea of Japan. National Museum of Nature and Science Monographs, 44, 225-291.

Sminkey, T.R. \& Tabit, C.R. (1992) Reproductive biology of the chain dogfish, Scyliorhinus retifer from the Mid-Atlantic Bight. Copeia, 1992 (1), 251-253.

Smith, H.M. (1907) The fishes of North Carolina. Vol. 2. E. M. Uzzell \& Co., Raleigh, 453pp.

Smith, H.M. (1912) Description of a new notidanoid shark from the Philippine islands, representing a new family. Proc. U.S. Natn. Mus., 41, 489-491.

Smith, J.L.B. (1937) New records of South African fishes. Natal Museum, 8 (2), 167-197.

Smith, J.L.B. (1949) The sea fishes of southern Africa. Central News Agency, Ltd., Cape Town, South Africa, 550pp.

Soares, K.D.A, Gadig, O.B.F. \& Gomes, U.L. (2015) Scyliorhinus ugoi, a new species of catshark from Brazil (Chondrichthyes: Carcharhiniformes: Scyliorhinidae). Zootaxa, 3937 (2), 347-361.

Soares, K.D.A, Gomes, U.L. \& de Carvalho, M.R. (2016) Taxonomic review of catsharks of the Scyliorhinus haeckelii group, with the description of a new species (Chondrichthyes: Carcharhiniformes: Scyliorhinidae). Zootaxa, 4066 (5), 501-534.

Sokhal, R.R. \& Rohlf, F.J. (1981). Biometry. Freeman, San Francisco.

Soldo, A., Dulcic, J. \& Cetinic, P. (2000) Contribution to the study of the morphology of the teeth of the nursehound Scyliorhinus stellaris (Chondrichthyes: Scyliorhinidae). Scientia Marina, 64 (3), 355-356. 
Soto, J.M.R. (2001) Annotated systematic checklist and bibliography of the coastal and oceanic fauna of Brazil. I. Sharks. Mare Magnum, 1 (1), 51-120.

Springer, S. (1966) A review of Western Atlantic cat sharks, Scyliorhinidae, with descriptions of a new genus and five new species. Fish. Bull. U S. Fish Wildl. Serv. 65 (3), 581-624.

Springer, S. (1973) Scyliorhinidae. In: Hureau, J.C. \& Monod, T. (Eds.), Check-list of the fishes of the north-eastern Atlantic and of the Mediterranean, UNESCO, Paris, 1, 19-21

Springer, S. (1979) A revision of the catsharks, Family Scyliorhinidae. NOAA Tech. Rep. NMFS 422, 97pp.

Springer, S. \& Bullis, H.R. (1956) Collections by the Oregon in the Gulf of Mexico. U.S. Fish Wildl. Serv., Spec. Sci. Rep. Fish., 196, 134pp.

Springer, V.G. \& Garrick, J.A.F. (1964) A survey of vertebral numbers in sharks. Proc. U.S. Nat. Mus., 116, 73-96.

Springer, S. \& Sadowsky, V. (1970) Subspecies of the western Atlantic catshark, Scyliorhinus retifer. Proc. Biol. Soc. Wash. 83 (7), 83-98.

Sumpter, J.P., Jenkins, N. \& Dodd, J.M. (1978) Gonadotrophic hormone in the pituitary gland of the dogfish (Scyliorhinus canicula L): distribution and physiological significance. General and Comparative Endocrinology, 36 (2), 275-285.

Swainson, W. (1839) On the natural history of fishes, amphibians, and reptiles. Longman, Orme, Brown, Green \& Longmans, London, 452pp.

Tanaka, S. (1908) Notes on some Japanese fishes, with descriptions of fourteen new species. $J$. Coll. Sci., Imp. Univ. Tokyo, 23, 1-54.

Tanaka, S. (1909) Descriptions of one new genus and ten new species of Japanese fishes. J. Coll. Sci., Imp. Univ. Tokyo, 23, 1-27. 
Tanaka, S. (1911) Figures and descriptions of the fishes of Japan. Daichi Shoin, Tokyo, 1, 118.

Tanaka, S. (1931a). On the distribution of fishes in Japanese waters. J. Fac. Sci., Tokyo Imp. Univ., 4, 3, 1-90.

Thompson, W. (1856) Fishes of Ireland. In: The natural history of Ireland. Vol. IV. Mammalia, reptiles, and fishes, also invertebrata. Fishes of Ireland. London, 69-268.

Tortonese, E. (1956) Fauna d'Italia. Vol. 2. Leptocardia, Cicrostomata, Selachii. Edizioni Calderini, Bologna, 327pp.

Ueno, T. (1971). List of marine fishes from the waters of Hokkaido and its adjacent regions. Sci. Rep. Hokkaido Fish. Exp. St. (13), 61-102.

Vaillant, L.L. (1888) Expeditions scientifiques du "Travailleur" et "Talisman" pendant les annees 1880, 1881, 1882, 1883. Poissons. G. Masson, Paris, 406pp.

Valmont, J. C. B. (1769) Dictionnaire raissoné d' histoire naturelle. Vols. 10 e 12.538pp.

Van der Laan, R., Eschmeyer, W.N. \& Fricke, R. (2014) Family-group names of Recent fishes. Zootaxa Monograph, 3882 (1), 1-230.

Weibezahn, F. H. (1953) Una nueva especie de Scyliorhinus de Venezuela. Novedades Científicas del Mus. Hist. Nat. La Salle, series Zool. 9: 1-7.

Weigmann, S. (2016) Annotated checklist of the living sharks, batoids and chimaeras (Chondrichthyes) of the world, with a focus on biogeographical diversity. Journal of Fish Biology, 88 (3), 837-1037.

Wetherbee, B.M., Gruber, S.H. \& Cortes, E. (1990) Diet, feeding habits, digestion and consumption in sharks, with special reference to lemon shark, Negaprion brevirostris. In: Pratt, H.L., Gruber, S.H. \& Taniuchi, T. (Eds.), Elasmobranchs as living resources: 
advances in the biology, ecology, systematics, and the status of the fisheries. NOAA Technical Report NMFS, 90, 29-47.

Wheeler, A. (1969) The fishes of the British Isles and north-west Europe. Macmillan, London, $613 \mathrm{pp}$.

White, E.G (1936a) A classification and phylogeny of the elasmobranch fishes. American Museum Novitates, 837, 1-16.

White, E.G. (1936b) Some transitional elasmobranchs connecting the catuloidea with the carcharinoidea. American Museum of Natural History, 879, 1-21.

White, E.G. (1937) Interrelationships of the elasmobranchs with a key to the order Galea. Bulletin of the American Museum of Natural History 74 (2), 25-138.

Whitley, G.P. (1939) Taxonomic notes on sharks and rays. Aust. Zool., 9, 227-262.

Wirtz, P., Brito, A., Falcón, J.M., Freitas, R., Fricke, R., Monteiro, V., Reiner, F. \& Tariche, O. (2013) The coastal fishes of the Cape Verde Islands - new records and an annotated check-list (Pisces). Spixiana, 36 (1), 113-142.

Yamada, U. (1986) Scyliorhinidae, Squalidae. In: Okamura, O. (Ed.), Fishes of the East China Sea and the Yellow Sea. Seikai Regional Fisheries Research Laboratory, Nagasaki, pp. 825 .

Yapp, W.B. (1958) Manual of elementary Zoology. $13^{\mathrm{a}}$ ed. Oxford University Press, London, 769pp. 